\title{
MEDICAL MANAGEMENT OF A SURVIVOR OF SEXUAL ASSAULT/ABUSE
}

\author{
Including Post-Exposure Prophylaxis and the Forensic Kit
}

Adrienne Wulfsohn, $M B B C h$

Private Medical Practitioner

\section{MEDICAL MANAGEMENT}

\section{INTRODUCTION}

(a) It is important to remember that survivors of sexual assault are patients like any other patients but with special needs. The basics of good clinical medicine should therefore apply including good history taking, clinical examination, special investigations and appropriate management.

(b) Every patient should be seen and managed in a comfortable and compassionate environment.

(c) The appropriate Crime Kits for the medico-legal examination should be available in hospitals and doctors' rooms, as South African Police Service (SAPS) personnel do not always have easy, quick access to the kits. This is one of the many reasons why a positive and co-operative working relationship with the relevant local SAPS is vital.

(d) All health care staff should undergo basic training in counselling and should have been trained in general medico-legal procedures regarding rape/sexual assault.

\section{PROCEDURES}

(a) If the patient is a priority 1 (immediate life-threatening injury $=$ code red) or priority 2 (limb/potential lifethreatening injury = code yellow) they should be managed in the resuscitation room according to Advanced Trauma Life Support protocols.

(b) If the patient is a priority 3 (non-limb or lifethreatening injury = code green) they should be treated in a dedicated, private room.

(c) A registered nurse and a medical doctor should commence by taking the medical history and details of the actual sexual assault.

(d) Once the history has been taken, the medical procedures that will be necessary should be explained to the patient in detail. (e) If the patient decides to lay a charge, the appropriate medico-legal examination should be explained in detail.

(f) If the patient is treated in a hospital, all injuries should be attended to, and patient transferral should be kept to a minimum.

(g) However, referrals may be necessary in certain circumstances, e.g. a child with vaginal tears requiring suturing under general anesthesia by a specialist surgeon.

(h) If the patient requires special investigations, e.g. x-rays, or requires admission for specialised medical treatment, this should be carried out.

(i) Where appropriate antibiotic prophylaxis should be commenced, as soon as possible.

(j) If possible, a facility should be made available where the patient can shower/bath and change their clothing on completion of the full medico-legal examination.

(k) Follow-up examinations should take place at 6 weeks, 3 months and 6 months after the assault. The choice of whether to attend for regular annual follow-up visits should be left to the patient.

\section{COUNSELLING}

(a) A trained counsellor should be called in for every case of sexual assault. He or she would be in a position to assist the family, friends, spouse and victim throughout the entire procedure and then provide follow-up counselling on a regular basis, according to the patient's needs.

(b) The patient has a right to lay a charge against the perpetrator(s). This should be facilitated with the relevant SAPS. Statistics from one large Johannesburg Hospital Trauma Unit show that less than $48 \%$ of patients are accompanied to casualty by a member of the SAPS. It may therefore be necessary to call in a SAPS officer for unaccompanied patients wishing to lay a charge. Data indicate that over $70 \%$ of sexual assault patients lay 
charges against the perpetrator(s), with appropriate treatment and explanations.

(c) Experience has shown that patients who see a counsellor at the initial event have improved follow-up rates for themselves and their families.

\section{POST-EXPOSURE PROPHYLAXIS (PEP)}

(a) A component of the initial history taking should include pre-test counselling for an HIV test.

(b) Consent for the management and procedures to be undertaken should be obtained.

(c) Informed consent for the baseline HIV enzyme-linked immunosorbent assay (ELISA) should be obtained. If the patient is under age the appropriate parent/guardian should be approached. If they are unavailable the attending SAPS officer will assist. If reliable rapid tests are available these may be used.

(d) The HIV ELISA results should be available within 3 hours.

(e) In circumstances where the results of conventional HIV ELISA tests may be delayed the use of rapid antibody tests is endorsed. A positive rapid test result would need to be confirmed by the use of a conventional ELISA test.

(f) Every patient should be told about PEP.

(g) If the ELISA test is negative, patients seen within 72 hours of the sexual assault should be offered antiretroviral (ARV) PEP. This should consist of a 28-day course of AZT and 3TC (Combivir), one tablet twice daily. Children and the elderly, who may find capsules difficult to swallow or require a lower dosage than provided by Combivir, can be given AZT/zidovudine (Retrovir) and 3TC/lamivudine syrup.

(h) Patients presenting more than 72 hours after the sexual assault should be counselled and offered routine blood testing, counselling and follow-up for HIV/AIDS and the rape incident.

(i) If the patient's HIV ELISA is positive, he or she should be counselled and referred to relevant centres for HIV-AIDS treatment.

(j) A pregnancy test should be done if necessary.

(k) Adherence to PEP does not appear to be problematical among survivors of sexual assault.

(I) The follow-up rate is excellent within the first 3 months and may only decline thereafter.

(m) The unit should provide patient follow-up for other medical problems, as well as counselling.

\section{OTHER CONCOMITANTLY PRESCRIBED MEDICATION}

Prophylactic antibiotics. In addition to the ARVs the patients should be given prophylactic antibiotics for other sexually transmitted infections:

- Penicillin 2 million units intramuscularly (IM) OR

Ciprobay $2 \mathrm{~g}$ stat.

- Flagyl 2 g p.o. stat

OR

Flagyl 500 mg intravenously (IV) should the patient be vomiting or nauseous.

Zithromax 1 g p.o. stat

OR

Tetracyclines $500 \mathrm{mg}$ q.i.d. for 10 days.

Patients who are allergic to penicillin should be given erythromycin.

\section{Other medication to consider:}

- The 'morning after' pill should be given if appropriate and necessary. This should be given at least 1 hour before giving the antibiotic prophylaxis.

- A vaginal douche should be provided to take home.

- Anti-emetics should be prescribed if necessary.

- Analgesia/anti-inflammatory medication should be prescribed if necessary.

\section{THE FORENSIC KIT — DIRECTIONS FOR USE}

The following steps should help you to understand the kit and make it easier to work with.

\section{GENERAL}

(a) It is imperative that from the commencement to the completion of the medical examination, a correct chain of events (evidence) is adhered to.

(b) It is therefore desirable that a second health care professional is present during the course of the forensic examination.

\section{THE CRIME KIT}

The new Crime Kit consists of:

1. The Evidence Collection Form and

2. The Crime Kit itself, which contains 7 sections. (Not all 7 sections will necessarily be used in all rape cases.)

\section{The Evidence Collection Form}

(a) The form (1 page) needs to be completed in triplicate.

(b) The first section of the document requests all of the particulars of the examining doctor. 
(c) The second section asks the patient when he/she last had consensual intercourse. It is vital that this question be asked, although it may prove difficult, because if consensual intercourse has taken place within 5 - 7 days before the assault semen from the consenting partner may still be present. This can make the forensic examination difficult to interpret.

(d) Should consensual intercourse have taken place within 5 - 7 days before the assault the justice system may request a blood sample, for DNA testing of the initial consenting partner, prior to going to court

(e) The third section requests all of the patient's details.

This is a vital part of the form that must be completed, as it is the only piece of documentation showing the patient's name and details, since these cannot be reflected on the outside of the forensic collection box.

(f) The fourth section requests the doctor or examiner to list the specimens that have been taken.

(g) The doctor and patient both need to sign the form.

(h) The first copy of the form should be placed in the Crime Kit box.

(i) The doctor retains the second copy in the patient's file for medico-legal purposes should the case go to court.

(j) The third copy, together with the forensic evidence bag, is handed to the member of the Family Crime and Sexual Violence Unit (FCS) unit or SAPS unit who collects the kit.

\section{THE CRIME KIT}

\section{A. Section 1. The oral swab}

This is used to detect any seminal fluid present in the buccal cavity if the patient has been forced to perform oral sex.

The kit contains the following:

- A swab box

- A swab

An evidence seal.

NB: the swab must not be moistened.

\section{Procedure:}

Swab the following areas with the same swab:

Under the tongue

- Along the inner, outer, upper and lower gum line

- The cheek

The palate.

There are extra swabs if necessary. Then:

- Place the swab in the box

- Fold as instructed

- Seal with an evidence seal.

\section{B. Section 2. Collection of underwear}

The kit contains the following:

A brown paper bag for the panties (or panties and/or pad) worn during or immediately after the assault. NB: sanitary pads attached to panties should be left attached.

\section{Procedure:}

The instructions request:

- Collection of the patient's panties and/or sanitary pad that she may have been wearing during or immediately after the assault.

- If the pad is separate from the panties, cover the adhesive (outside) side of the pad with the wax strip provided.

- If the victim is a man or boy, the underpants must be collected.

- Place all items in the bag.

- Seal the bag by removing the self-adhesive backing from the bag.

- Place the evidence sticker where the lip and the body of the bag meet.

- If the patient was wearing a tampon, see Section 7 (Tampon collection).

C. Section 3. Collection of other evidence from the patient's body

This section provides for the correct removal of evidence from the patient's body.

There are two steps (C.1 and C.2) to this part of the kit.

C.1. Collection of evidence from the patient's body, e.g. foreign debris and/or dried secretions

The kit contains the following:

- Sterile swabs

- Sharp-pointed sterile swabs (2)

- Sterile water

Ruler

Box for swabs

- A catch paper for reference samples.

\section{Procedure:}

- Look for any areas on the body on which there may be dried secretions, and also ask the patient if they feel any area(s) of dried and/or caked secretions on their body.

- To identify secretions, ask the patient if she/he knows where they might be. Ask the patient if the perpetrator kissed, licked or bit her/him anywhere. Those areas should be swabbed.

- To prevent destroying any evidence, one evidence source should be swabbed at a single time. 
This means one 'stain' per swab. This is to prevent mixing evidence if there was more than one attacker.

- The swab should be moistened with the sterile water provided before swabbing the body area.

- If the patient reports having scratched the perpetrator fingernail scrapings must be taken. Before swabbing under the fingernail(s) loosen the debris with a sterile blade (2 swabs with sharp points are provided in the kit) which can be used to swab under the nail(s). The swab must be moistened with the sterile water provided.

- Any bruise(s)/bite mark(s) should be measured with the ruler provided.

- Pack the swabs in the box provided.

Break off the ends and close with an evidence seal.

\section{C.2. Collection of evidence from the patient's hair}

The kit contains the following:

- A comb

- A catch paper for the head combings

- A catch paper for reference samples.

\section{Hair combing procedure:}

- Place the catch paper under the patient's head.

- Comb the hair so that any loose hair or debris fall onto the catch paper marked 'head hair combing'. Ensure that there are sufficient samples.

- Comb hair in a downward direction to ensure that any foreign debris falls onto the catchment paper.

You may use your fingers to ruffle the hair to loosen any debris that may be stuck in the hair.

Fold the paper with the comb inside it .

- Place in pack.

- Seal the pack with an evidence sticker.

Reference head hair:

- Pull at least 5 hairs from the top, back, front, left and right side of the head.

- Place the hair on the catch paper marked pulled 'reference head hair'.

Fold the paper and seal with an evidence seal.

D. Section 4. Collection of debris that will link the patient to the location of the assault

The kit contains the following:

- Catch paper marked 'debris A' for matted hair from the head or body which might indicate blood/semen

- Catch paper marked 'debris B' for debris taken from the body.

Procedure for collecting samples:

Debris A:

Look for matted hair on the head and/or body that may indicate blood/semen.

- Cut the hair over the catch paper marked 'debris A'.
Fold the paper and seal with the evidence seal.

\section{Debris B:}

Collect any debris such as soil, leaves, grass and hair that may be present during the examination.

- Use a spatula and remove the debris from the patient's body.

- Place it on the catch paper marked 'debris B'.

Fold the paper.

- Seal with the evidence seal.

E. Section 5. Collection of pubic hair and reference hair This is done to attempt to identify the perpetrator. This section contains two sub-sections: E1 for samples of pubic hair and E2 for reference hair.

The kit contains the following:

- A comb

- Catch paper marked 'pubic hair combing'

- Catch paper marked 'reference pubic hair'.

\section{E.1. Pubic hair combing}

Procedure for collecting pubic hair combings:

- Place the catch paper marked 'pubic hair combing' under the patient's buttocks.

- Comb the hair in a downward direction over the catch paper.

- Comb with your fingers to assist in loosening debris

- If matted hair is noted, cut it off over the catch paper.

- Fold the paper.

- Seal with evidence seal.

\section{E.2. Reference hair}

Procedure for collecting reference pubic hair combings:

- This is needed for microscopic screening

With the patient's consent, pull out about 10 pubic hairs.

- Place the hair into the catch paper marked 'reference pubic hair'.

- Fold.

- Seal with the evidence seal.

F. Section 6. Collection of anal samples (used in anal rape)

NB. The ano-rectal examination should be carried out before the vaginal examination to prevent crosscontamination of evidence.

The kit contains the following:

- Two swabs

- An amp of sterile water

Racks marked 'A' and 'B'. 
Procedure for collecting samples from the external anal area and the rectum:

\section{External anal area:}

- Put the patient into a comfortable position, e.g. left lateral position.

Moisten the swab with the sterile water provided.

- Swab the external anal area, extending slightly into the anal canal.

Place the swab in the rack marked ' $A$ '.

Rectum:

- Apply gentle lateral tension to the peri-anal area for about 3 minutes to dilate the anal sphincter.

- After dilatation has taken place, use one swab to swab the rectal canal.

- Place the swab in the rack marked 'B'.

- Fold pack.

- Close with evidence seal.

G. Section 7. Collection of vaginal swabs (used in vaginal rape)

The kit contains the following:

- Three swabs

- An amp of sterile water

- Racks marked 'A', 'B' and 'C'

- Box marked 'Tampon'.

Procedure for collecting vaginal biological material for DNA testing that may identify the perpetrator: (NB: Swabs are taken of three areas: external genital area, vagina and cervix).

\section{External genital area swab:}

Moisten the swab with the sterile water provided.

- Swab the external and internal surface of the labia majora and minora including the clitoris, peri-urethral area and fosse navicularis.

- Place the swab in the rack marked 'A'.

Vaginal swab:

- This must be done before the internal clinical examination.

- Insert an unlubricated speculum

- Swab the anterior and posterior vaginal fornices

- Place the swab in the rack marked 'B'.
Cervical swab:

- Swab the inside of the cervical os.

- Collect as much of the mucous plug as possible.

- Place the swab in the rack marked ' $C$ '.

- Fold.

- Seal the box with the evidence seal.

- Place sellotape around the box.

\section{Tampon collection:}

If a tampon was present during the rape or inserted after the rape, it should be retained for evidence. The procedure for collection is:

Remove the tampon

- Place in the box marked 'Tampon'

- Seal the box with the evidence seal.

\section{Additional items}

Collection of reference DNA specimen - which need not be included in the kit.

- This provides the forensic laboratory with a sample of the patient's blood.

Once the blood has been taken and put in the purplecapped tube, insert the cannula provided.

- Press the cannula onto the cassette. Ony one drop of blood is needed.

- Place the cassette in the bag provided.

- Seal with an evidence seal.

\section{Box closure}

Once all the specimens have been taken, place them in the box.

- Leave the unused packs in the box.

The front page of the evidence collection form is placed on top of the specimens.

On the outside of the box is a space to write the police station and case number.

- There are three stickers with numbers on, on the upper left corner of the box.

One sticker goes onto the J88 form.

- The second sticker goes onto the retained copy of the form which is stapled onto the inside cover of the patient file.

- The third sticker stays on the box.

- The box is then sealed with the evidence sticker provided.

- Place sellotape around the box to keep it closed.

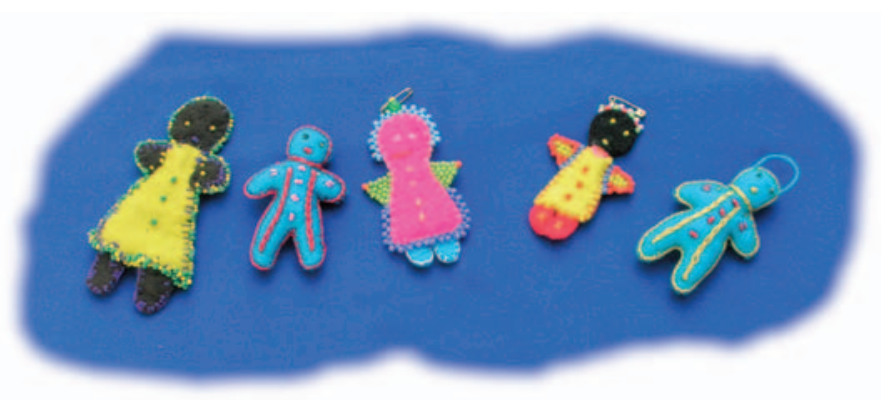

